\title{
EXCESS ANALYTE DISPERSION GENERATED BY TURNS ON MICROCHIPS
}

\author{
Christopher T. Culbertson, Stephen C. Jacobson, and J. Michael Ramsey \\ Oak Ridge National Laboratory, P.O. Box 2008 \\ Oak Ridge, TN 37831-6142
}

\begin{abstract}
Introducing turns into the separation channel on a microchip device generally introduces an additional geometrical contribution to analyte dispersion. A simple, one-dimensional model has been developed which predicts the amount of excess dispersion introduced by turns in microchip channels. This model accounts for migration length differences, field strength differences, and transverse diffusion effects. The model describes the experimental data well.
\end{abstract}

\section{INTRODUCTION}

Microfluidic devices have been successfully demonstrated for a wide variety of separation techniques including capillary electrophoresis(CE), [1-3] synchronized cyclic electrophoresis(SCE),[4] free-flow electrophoresis, [5] and gel electrophoresis(GE).[6, 7] There has been a concerted effort to integrate these separation techniques with other physical manipulations and chemical reactions on monolithic microchip devices[8-12] as the advantages of combining sample processing and analysis in a single device are numerous. This increased level of integration, however, is reflected in a more complex channel manifold with a greater number of channels and interconnections. To maintain the overall small compact footprint of the microchip the channel manifold has to be carefully designed. Often these designs require that several turns be introduced into various channels, including the separation channel which in general has the greatest channel length requirements. [3]

Unfortunately, the introduction of turns into the separation channel also introduces a potential source of analyte dispersion.[3] This potential source of dispersion is introduced as a consequence of the differences in the arc length and field strength across the channel width in the turns (Figure 1A). As an analyte band traverses a turn, the individual molecules in the band will migrate different distances around the turn and feel different electric field strengths depending upon their respective positions across the width of the channel. This can result in an elongation of the band (Figure 1B). We have developed a model to describe the dispersion generated by a turn which accounts for the travel distance and the field strength differences experienced by molecules as they transit the turn and includes the effects of transverse diffusion. This model predicts that the variance introduced by a turn will be given by equation 1 below:

$$
\sigma_{t u r n}^{2}=\frac{\left(2 \theta w\left(1-\exp \left(-t_{D} / t_{t}\right)\right)\right)^{2}}{24}
$$

where $\theta$ is the angle subtended by a turn, $w$ is the channel width, $t_{D}$ is the analyte transverse diffusion time, and $t_{t}$ is the time that an analyte spends traversing the turn. The constant in the denominator is determined from the input response function which generated the variance.

Using this model the contribution of the geometrically generated dispersion to the total peak variance is examined as a function of the ratio of the transverse diffusion time to the analyte turn transit time $\left(t_{d} / t_{t}\right)$.
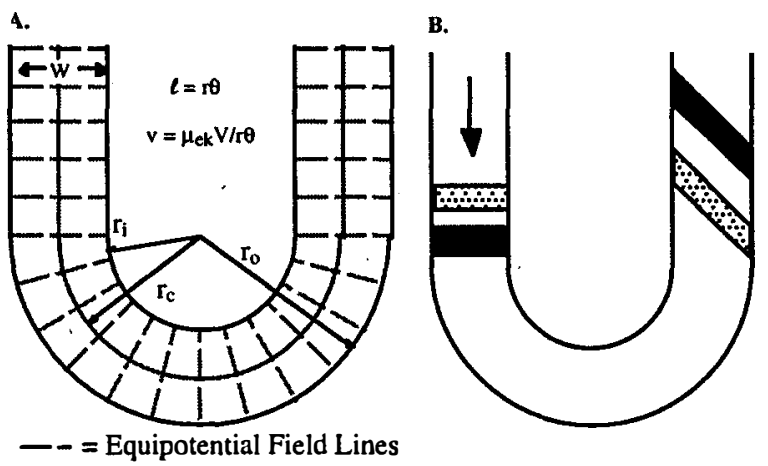

Figure 1. A. The distance ( $(\ell)$ that an analyte molecule travels around a turn and the field strength experienced in a turn depend upon the molecule's radial position. $B$. When the diffusion time across the channel is slow compared to the transit time around the turn $\left(t_{D} / t_{t}\right.$ large $)$ then excess dispersion is introduced by the turn as the molecules situated along the inside of the turn move more quickly through the turn than those along the outside.

\section{EXPERIMENTAL}

Chip Design. The general design of the microchips used in this study can be found in Figure 2. They were fabricated from soda-lime glass slides using standard photolithographic, wet chemical etching, and cover plate bonding techniques.[3]

Apparatus. All experiments were performed using a single point, laser induced fluorescence (LIF) detection system similar to that previously described.[10] The $514 \mathrm{~nm}$ laser line was focused through two cylindrical lenses to create an ellipsoidal excitation spot - the long axis of the ellipse being orthogonal to the channel axis. The fluorescence was collected using a $20 x$ microscope objective and was focused onto a $150 \mu \mathrm{m}$ wide air slit. This slit was long enough to sample the entire width of the separation channel. The signal passing through the slit was

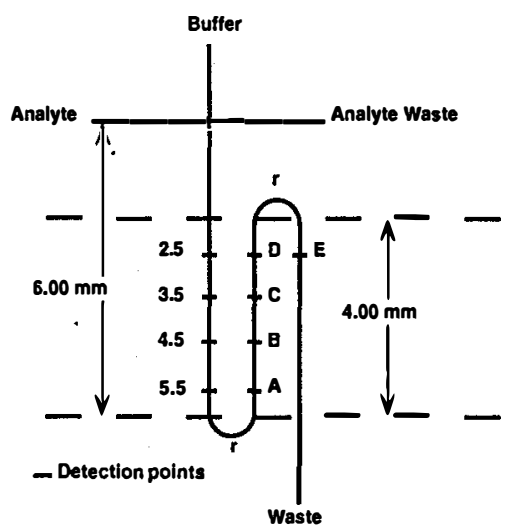

Figure 2. Schematic diagram of chip geometry. 
spectrally filtered using a bandpass filter (560df40; Omega). before being measured at a photomultiplier tube (PMT, Oriel \#77340). The electrophoretic separations and injections were performed using four independent and remotely programmable high voltage (0-10 kV) power supplies (Ultravolt 10A12-P4). Experimental protocol. Electrophoretic injections of all analytes were performed in the pinched (constant volume) mode.[3] Each experimental sequence consisted of 2 or 3 replicate runs at several detection points (Figure 2). A plot of the peak variance versus migration time for one of these experimental sequences allows the quantitation of all of the major analyte band variance sources on the chip.

\section{RESULTS AND DISCUSSION}

To determine the robustness of the model developed above for predicting the magnitude of the geometrical dispersion introduced by turns, a wide range of $t_{D} / t_{\text {, values }}$ were examined using a variety of chips and buffer conditions. A total of 37 experiments were performed. Each of these experiments consisted of approximately 30 electrophoretic runs. The actual variance introduced by the turn was determined using the equation below

$$
\sigma_{\text {turm. act }}^{2}=\sigma_{A}^{2}-\sigma_{5.5 \mathrm{~mm}}^{2}-2 * D *\left(t_{A}-t_{5.5 \mathrm{~mm}}\right)
$$

where $\sigma_{A}^{2}$ is the peak variance at detection point $A$ after the first turn (see Figure 2), $\sigma_{5.5 \mathrm{~mm}}^{2}$ is the peak variance at the 5.5 mm detection point just prior to the turn, $D$ is the analyte diffusion coefficient, $t_{A}$ is the migration time for the analyte at point $\mathrm{A}$, and $t_{5.5 \mathrm{~mm}}$ is the migration time of the analyte at the $5.5 \mathrm{~mm}$ detection point. The actual variances were then normalized using $(2 \theta w)^{2}$ and plotted against the $t_{D} / t_{\text {t }}$ ratio as shown in Figure 3. Eleven chips of varying channel widths

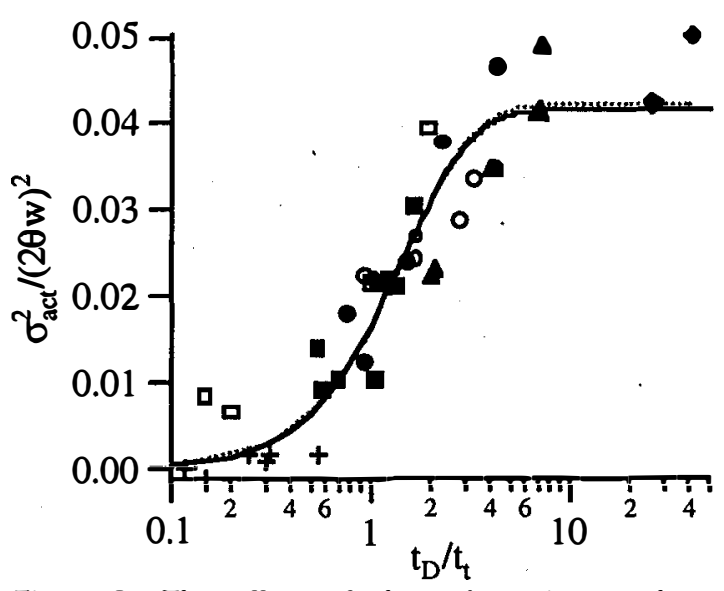

Figure 3. The effect of the $t_{D} / t_{1}$ ratio on the excess dispersion introduced by a turn. The dotted line shows the best fit to the data. The solid line is that predicted by equation 1

and radii of curvature were used in collecting the data points shown. The channels on the chips ranged from 38 to $60 \mu \mathrm{m}$ in width at the top of the channel. The turns on the chips had radii of curvature of $125 \mu \mathrm{m}(\Delta, \diamond), 250 \mu \mathrm{m}(\bullet, 0)$, or 500 $\mu \mathrm{m}(\boldsymbol{\square}, \square)$. The analytes and buffer systems consisted of
Rhodamine B in a 50/50 (v/v) $\mathrm{MeOH} / 20 \mathrm{mM} \mathrm{NaBorate}$ buffer $(\triangle, \bigcirc, \square)$, TRITC-Arg in a $10 \mathrm{mM} \mathrm{NaBorate}$ buffer $(O, \square)$, and a restriction digest of $\phi \times 174$ in a gel filled capillary with a $0.5 \mathrm{x}$ TBE buffer $(\diamond)$. The analyte velocities varied from $0.0051 \mathrm{~cm} / \mathrm{sec}$ to $0.212 \mathrm{~cm} / \mathrm{sec}$. The results are consistent with the model.

\section{ACKNOWLEDGMENTS}

This research is sponsored by The Office of Research and Development, US Department of Energy, under contract DE-AC05-96OR22464 with Oak Ridge National Laboratory, managed by Lockheed Martin Energy Research Corporation. C.T.C. was supported through an appointment to the ORNL Postdoctoral Research Associates Program administered jointly by ORNL and the ORISE.

\section{REFERENCES}

1. Harrison, D.J., et al., Micromachining a Miniturized Capillary Electrophoresis-Based Chemical Analysis System on a Chip. Science, 1993. 261: p. 895-897.

2. Effenhauser, C.S., A. Manz, and H.M. Widmer, Glass Chips for High-Speed Capillary Electrophoresis Separations with Submicrometer Plate Heights. Analytical Chemistry, 1993. 65(19): p. 2637-2642.

3. Jacobson, S.C., et al., Effects of Injection Schemes and Column Geometry on the Performance of Microchip Electrophoresis Devices. Analytical Chemistry, 1994. 66(7): p. 1107-1113.

4. Burggraf, N., et al., Synchronized Cyclic Capillary Electrophoresis - A Novel Approach to Ion Separations in Solution. Journal of High Resolution Chromatography, 1993. 16: p. 594-596.

5. Raymond, D.C., A. Manz, and H.M. Widmer, Continuous Sample Pretreatment Using a Free-flow Electrophoresis Device Integrated onto a Silicon Chip. Analytical Chemistry, 1994. 66(18): p. 2858-2865.

6. Effenhauser, C.S., et al., High-Speed Separation of Antisense Oligonucleotides on a Micromachined Capillary Electrophoresis Device. Analytical Chemistry, 1994. 66(18): p. 2949-2953.

7. Woolley, A.T. and R.A. Mathies, Proceedings of the National Academy of Sciences USA, 1994. 91: p. 11348.

8. Jacobson, S.C., et al., Precolumn Reactions with Electrophoretic Analysis Integrated on a Microchip. Analytical Chemistry, 1994. 66(23): p. 4127-4132.

9. Fluri, K., et al., Integrated Capillary Electrophoresis Devices with an Efficient Postcolumn Reactor in Planar Quartz and Glass Chips. Analytical Chemistry, 1996. 68(23): p. 4285-4290.

10. Jacobson, S.C. and J.M. Ramsey, Integrated Microdevice for DNA Restriction Fragment Analysis. Analytical Chemistry, 1996. 68(5): p. 720-723.

11. Woolley, A.T., et al., Functional Integration of PCR Amplification and Capillary Electrophoresis in a Microfabricated DNA Analysis Device. Analytical Chemistry, 1996. 68(23): p. 4081-4086.

12. Waters, L.C., et al., Microchip Device for Cell Lysis, Multiplex PCR Amplification, and Electrophoretic Sizing. Analytical Chemistry, 1998. 70(1): p. 158-162. 